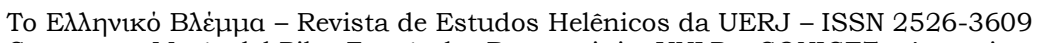
Separata - María del Pilar Fernández Deagustini - UNLP - CONICET - Argentina

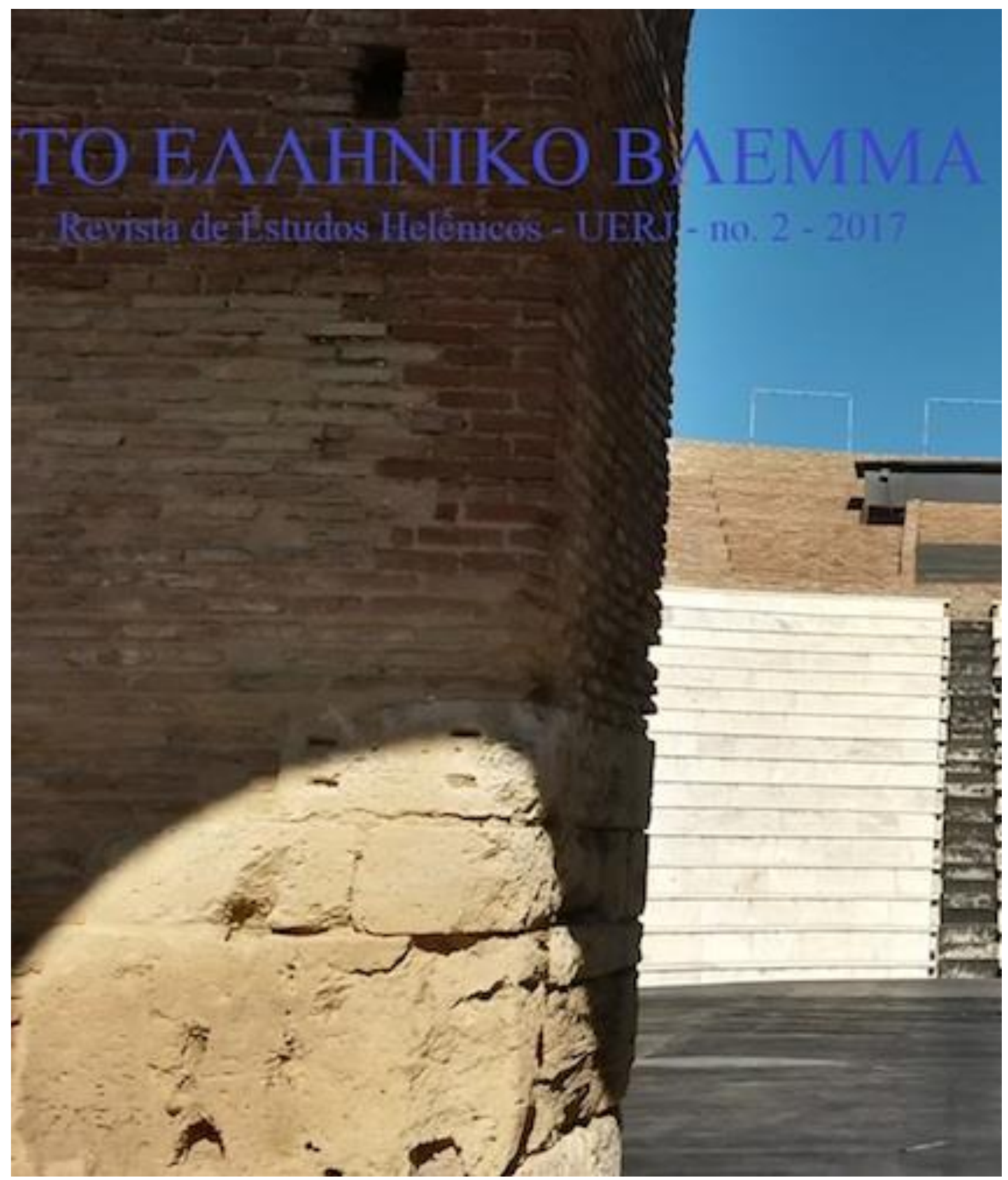




\title{
EL SENTIDO DE LA FORMA. \\ LOS 'ANUNCIOS EXTENDIDOS' EN SUPLICANTES DE ESQUILO'
}

\author{
María del Pilar Fernández Deagustini \\ UNLP- CONICET \\ mpilarfd@hotmail.com
}

"...it is up to the playwright to invent the stage action and to use it; the few fixed elements make no difference to this. And it is, indeed, precisely in his dramatization that his art lies; not in the story, but in how he turns it into the drama."

Taplin (1977: 27).

\section{Resumen}

Suplicantes de Esquilo fue datada y valorada como "primitiva" en nombre de un patrón genérico convencional. Frente al enfoque normalizador de origen aristotélico, artificial y forzado, proponemos apreciar la obra como un fenómeno dramático auténtico, conjugando el método filológico-literario con la perspectiva performativa, un enfoque escasamente ensayado en las aproximaciones a Suplicantes.

En el presente artículo, analizamos dos pasajes de la tragedia, los "anuncios extendidos" (vv. 176-203; 710-733), porque permiten revelar su técnica estructural auténtica, sin imponer a la obra una matriz compositiva externa. Nuestro objetivo es demostrar que la forma de la puesta en escena confiere sentido a la matriz discursiva.

Palabras clave: Sentido- Estructura- Anuncios- Suplicantes- Esquilo

\begin{abstract}
Aeschylus' Suppliants was dated and valued as "primitive" according to a generic and conventional pattern. Confronting this standarized approach of aristotelic origin, artificial and forced, we propose to esteem the play as an authentic dramatic phenomenon, combining the filological-literary method with the performative perspective, which is an unusual standpoint among the studies of Suppliants.

In this article, we analize two passages of the tragedy, the "extended announcements" (vv. 176-203; 710-733), because they allow for revealing its authentic estructural technique, without imposing an external scheme on the play. Our aim is to demonstrate that the form of the performance transmits meaning to the discursive pattern.
\end{abstract}

Key words: Meaning- Structure- Announcements- Suppliants- Aeschylus

Durante décadas, Suplicantes ha sido considerada la obra dramática más antigua entre las conservadas, hasta que, mediando el siglo pasado, nueva evidencia puso en cuestión su datación primera, junto con todo aquello que había sido escrito

\footnotetext{
${ }^{1}$ Parte de esta sección del apartado está constituido por el contenido de la ponencia "Cooperación y competencia: las llegadas del amigo y del enemigo en Suplicantes de Esquilo", presentada en el VI Coloquio Internacional Agón: Competencia y Cooperación. De la antigua Grecia a la Actualidad. Homenaje a Ana María González de Tobia, en la cuidad de La Plata, junio de 2012, inédito.
} 
con anterioridad al descubrimiento. ${ }^{2}$ A pesar de haber sido protagonista de uno de los más revolucionarios hallazgos de los estudios clásicos, Suplicantes es el drama menos estudiado entre las obras de Esquilo y uno de los menos leídos del corpus trágico atesorado. Entre los estudios existentes, menguan interpretaciones integrales de la tragedia que reconozcan su impronta genérica. Debido al desafortunado estado del manuscrito, los críticos concentraron su interés en aproximaciones de tipo textual y, como consecuencia de haber sido considerada el único exponente de la prototragedia, su valor dramático ha sido históricamente subestimado. ${ }^{3}$

Suplicantes fue datada y valorada como "primitiva" en nombre de un patrón genérico convencional, a partir del cual se determinó que la técnica dramática de la obra era arcaica (por carecer de prólogo y desarrollar un argumento "simple") y que las posibilidades ofrecidas por el uso del segundo actor no habian sido adecuadamente desarrolladas todavía, porque la lírica coral predominaba sobre el diálogo entre actores. Frente a este enfoque normalizador de origen aristotélico, artificial y forzado, proponemos apreciar la obra como un fenómeno dramático auténtico, conjugando el método filológico-literario con la perspectiva performativa, un enfoque escasamente ensayado en las aproximaciones a Suplicantes. El principio que guía este análisis es, entonces, que el entendimiento cabal del género trágico griego clásico sólo puede emerger del conocimiento de cuán diferentes entre sí son cada una de las obras que han subsistido hasta nuestros dias. A pesar de las limitaciones fijadas por las condiciones de la performance, cada drama es una obra de arte única, que obedece solamente a sus propias reglas. Por consiguiente, interpretar una tragedia particular implica intentar captar la concepción dramática subyacente, asumiendo que el dramaturgo compuso, podujo y dirigió la obra con absoluta libertad. Para ello, proponemos el análisis de dos pasajes, los "anuncios extendidos", ${ }^{4}$ para demostrar que el texto dramático es sólo uno de los insumos que construyen una experiencia, mucho más amplia y de inasequible reconstrucción: la composición de una "tragedia en acción" ${ }^{5}$ en la que la forma de la puesta en escena confiere sentido a la matriz discursiva.

En la década del '70, Taplin' ${ }^{6}$ supo probar que el estudio de la técnica estructural de la tragedia griega ha sido inhibido por los términos y definiciones de Poética 1452b 14-27. El acercamiento a la forma de una tragedia desde los axiomas aristotélicos propuestos en el conocido capítulo XII no sólo es inflexible y predeterminado, ${ }^{7}$ sino que se define sobre la base de un único criterio: la alternancia entre el discurso de los actores y los cantos del coro. En el caso particular de Suplicantes, la arbitrariedad del principio resulta evidente, puesto que el coro es protagonista. En una "tragedia lírica," donde el coro interviene extraordinariamente, participando en los "episodios", no todo canto coral divide escenas.

Sin dudas, la forma constituye un aspecto importante de cualquier obra de

\footnotetext{
2 El descubrimiento del papiro de Oxyrrinco 2256.3 en 1952 causó la mayor revolución en los estudios esquileos en particular y clásicos en general. El texto revelaba datos de un certamen trágico de las Grandes Dionisias que transformaron la historiografia del teatro griego clásico, sugiriendo una nueva fecha de representación de Suplicantes. El revolucionario fragmento, adjudicado a Aristófanes de Bizancio, fue publicado por primera vez por Lobel-WegenerRoberts (1952: 30). Tras siglos de recepción en los que se desconoció la fecha de representación de la obra, durante los cuales los especialistas se esforzaron por descifrarla, la publicación del papiro marcó un hito que dividió los estudios de la obra en dos etapas definidas. Antes de 1952, el desconocimiento de una fecha cierta impulsó el surgimiento de investigaciones que, basadas en criterios estilométricos e históricos, tendieron a ubicar la obra en una etapa temprana de la producción dramática de Esquilo, aproximadamente en la década del 490 a. C, dos décadas antes de la primera tragedia datada con certeza, Los persas (472 a. C.). Después de 1952, la divulgación de la corrompida didascalia impuso una nueva coyuntura entre los clasicistas, en la cual la mayoría se inclinó a sostener que Suplicantes no era la primera entre las tragedias conservadas de Esquilo ni, por lo tanto, la tragedia más antigua entre las preservadas de los tres trágicos.

Para una completa discusión del fragmento 2256.3, Garvie (2006: 1-28). Dos artículos específicamente dedicados a la polémica sobre la datación de Suplicantes son los de Yorke (1954) y Wolf (1958).

${ }^{3}$ Sobre los abordajes y líneas de investigación acerca de la obra, cfr. Fernández Deagustini (2015: "Introducción").

${ }^{4}$ El término para designar este tipo de pasajes dramáticos ha sido acuñado por Taplin (1977).

5 Se trata de una referencia explícita al emblemático libro de Taplin (1978).

${ }^{6}$ Cfr. Taplin (1977: 25 y 471-476).

7 Según el esquema aristotélico, las partes constitutivas de toda tragedia son prólogo, párodos, episodio, estásimo y éxodo.
} 
arte. En el caso particular del teatro, el dramaturgo no sólo compone cada una de las escenas como unidad, sino que las organiza significativamente, de manera que el espectador pueda responder a la relación de las partes en un todo integrado. En esta disposición de las distintas unidades dramáticas, dos pautas resultan fundamentales: por un lado, la secuencia, ${ }^{8}$ es decir, la concatenación ininterrumpida y progresiva de escenas; 9 por el otro, la coherencia, o sea, la existencia de componentes que aparecen en conjuntos solidarios y cooperan en la construcción del sentido dramático. En este último aspecto de la composición estructural, importan los momentos que captan el interés del espectador y orientan su apreciación de los hechos, por lo tanto, los momentos que tienen tanta prominencia como atención dedicada a ellos.

Proponemos enfocar la composición de Suplicantes desde este último aspecto, porque permite revelar su técnica estructural auténtica, sin imponer a la obra una matriz compositiva externa. Nuestra hipótesis es que los "anuncios extendidos" constituyen un recurso compositivo fundamental que da coherencia estructural a la presentación de la coyuntura trágica.

La limitación de los recursos técnicos disponibles para la composición de una tragedia griega clásica (cantidad de actores, de coreutas, tiempo de representación aproximado, entre muchos otros factores condicionantes) ofrecía comparativamente menos posibilidades de variedad, riqueza y complejidad que el teatro posterior. Sin embargo, los dramaturgos alcanzaron un notable grado de diversidad dentro de este marco escénico básico. ${ }^{10}$ Una de las premisas del enfoque performativo es que, cuanto más significativa es la acción de una tragedia, mayor es la atención que recibe en las palabras. ${ }^{11}$ Este argumento elemental indica, en el caso particular de Suplicantes, dos acciones deliberadamente señaladas como relevantes a partir del discurso: la llegada de Pelasgo y la del heraldo a las inmediaciones del altar donde se encuentra el coro de Danaides. En ambos casos, el ingreso a escena de los actores no se gesta a partir de una simple mención por parte de otro personaje en escena, sino de un vasto anuncio de Dánao, que captura la atención de las Danaides tanto como la del espectador. ${ }^{12}$ Debido a su extensión, los versos comprendidos entre 176203 y 710-733 son únicos en el corpus de las tragedias conservadas. ${ }^{13}$

La extraordinaria extensión de los pasajes que informan sobre estas dos llegadas no es la única particularidad que señala la trascendencia dramática de dichas acciones. En primer lugar, es llamativa la similitud contextual entre ambas: intervienen los mismos caracteres (Dánao y las Danaides) para reaccionar ante el

\footnotetext{
${ }^{8}$ Como sostiene Taplin (1977: 18), "the play should be treated as sequential; that is to say that, since the work was performed from start to finish in a certain time, it must be taken in order, and we should be wary of treating the play as 'spatial', that is as an indivisible whole in which all parts bear on all others".

${ }^{9} \mathrm{La}$ concatenación de escenas es ininterrumpida porque la tragedia griega tiene una continuidad formal peculiar dada por la continua presencia del coro.

10 Taplin (1977: 19-21) brinda un somero informe de ese marco, a partir del cual logra demostrar su carácter sumamente flexible.

11 Taplin (1977: 13).

12 Taplin (1977: 199): "As Danaus has actually seen the approach of the Argives the entire scene from 176 to 233 is strictly speaking an entrance announcement, according to the definition on p. 71 , rather than simply preparation. However, since one normally uses 'announcement' to refer to the familiar brief introductions devoted exclusively to the immediate arrival, and since most of this scene is spent, rather, on the response of the Danaids, the term is only loosely appropriate".

13 Taplin (1977: 200) destaca el carácter extraordinario de estos anuncios, pero sorprende el hecho de que explique el empleo de este recurso en Suplicantes como un resabio arcaico del teatro anterior a la skené, en el que todos los ingresos debían hacerse a través de los eísodoi. La justificación es insuficiente, ya que se basa en el repetido lugar de la crítica que tiende a ver los elementos arcaicos o primitivos de la obra. Por el contrario, el doble uso del anuncio extendido puede comprenderse a partir de la influencia del Altarmotiv, no sólo en relación con los elementos esenciales de ese tipo de trama, sino con las actividades esénicas que determina. Las reglas de la hiketeia obligan a todo suplicante a llevar a cabo determinados comportamientos. En acuerdo con la escena tipo de la súplica, la dilatación temporal que ofrecen los anuncios da tiempo escénico a las Danaides para ubicarse en el lugar que les corresponde en su carácter de suplicantes y tomar la posición que deben: postrarse a los pies de las estatuas con los ramos en sus manos. Además, las noticias del salvador y del raptor movilizan actitudes y movimientos gestuales opuestos: la postración silenciosa cede el lugar a la corrida veloz y el grito despavorido, en busca de un socorro que parece dificil de precisar.
} 
mismo suceso (la aproximación de un sujeto al recinto sagrado). En segundo lugar, el análisis filológico-literario confirma la relación de ambos pasajes en el nivel discursivo, en el que se manifiestan numerosos ecos léxicos y sintácticos. Finalmente, la afinidad entre las dos escenas se consolida en la esfera visual, dado que la representación de los discursos es idéntica: desde su posición privilegiada en lo alto del altar y tras un tiempo extenso de vigilar el horizonte, el actor que representa a Dánao interrumpe su largo silencio para comunicar al coro, que permanece en la orchéstra, la novedad de que alguien se aproxima a su encuentro.

La coincidencia en los aspectos contextual, textual y paratextual determina una conexión entre escenas que no puede ser ignorada. Durante la performance, el espectador de Suplicantes es expuesto dos veces a un cuadro escénico prácticamente análogo, revelando un patrón compositivo. Entre los recursos disponibles para el dramaturgo, la composición de escenas "en espejo" era una de las alternativas para que dos eventos separados cronológicamente pudieran ser percibidos en conjunto. Sea o no verdad que la mente humana tiene una base de operación binaria, sea cierto o no que la mente griega tenía una tendencia especial a ordenar el mundo en términos de polaridad y analogía, efectivamente los trágicos griegos componian escenas de a pares. ${ }^{14}$ Pero es necesario tener presente que, en este tipo de composición estructural, el público estaba en condiciones de percibir la trascendencia de los sucesos espejados y concebirlos como un todo solidario una vez materializada la segunda de ambas escenas. Ante la repetición, se habría generado una suerte de reconsideración y reevaluación de la situación primera, mientras se percibían las diferencias con la escena doble que se estaba representando. Por lo tanto, en la reflexión acerca de la performance de Suplicantes, no hay que perder de vista la envergadura dramática que cobraba el segundo anuncio extendido, por dos razones: la correlación con el primer anuncio y la introducción inesperada de la peripecia. ${ }^{15}$

La técnica estructural demuestra la relevancia de los pasajes que anuncian las llegadas del potencial amigo (phílos) y enemigo (ekhthrós) de las Danaides, los sucesos más significativos para el coro protagonista. Además, la esperanza del refugio primero y, más tarde, el presagio de la amenaza inminente constituyen los momentos más dinámicos del drama, porque ofrecen al espectador la oportunidad de percibir la impulsiva y apasionada reacción de las jóvenes ante los dos eventos que determinan su destino. Los anuncios de la llegada de los huéspedes Argivos y de los perseguidores Egipcios, por lo tanto, construyen las instancias de mayor clímax de la obra. Teniendo en cuenta estas características específicas de Suplicantes, el análisis filológicoliterario de ambos anuncios extendidos surge como un material provechoso para refutar el prejuicio de que se trata de una tragedia primitiva.

El examen comparativo de los dos anuncios extendidos revela cantidad de detalles que prueban no sólo su correlación verbal, sino también su dominio sobre la simetría y equilibrio compositivo de la tragedia. El primer discurso (176-203) sucede inmediatamente después de la oda inicial (1-175), es decir, del arribo de las Danaides a la costa de Argos, escapando del acoso violento de sus primos. Dice Dánao:

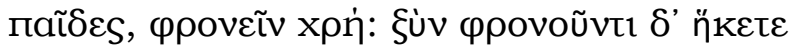

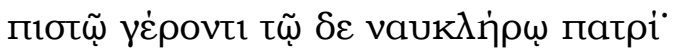

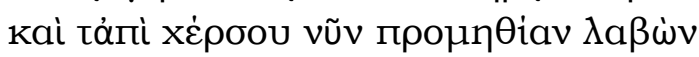

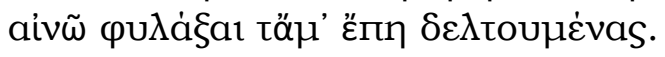

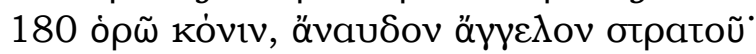

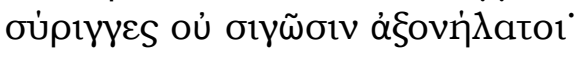

Hijas, es necesario ser sensatos. Pero llegáis con este sensato anciano digno de confianza, con vuestro padre, piloto de la nave. También ahora, sobre esta tierra seca, después de tomar una precaución,

\footnotetext{
14 Taplin (1978: 98).

15 Precisamente, Taplin (1977: 98) advierte que la tendencia a un giro catastrófico central fomentó la composición a través de escenas dobles en la tragedia griega clásica, una en cada mitad de la trama.
} 


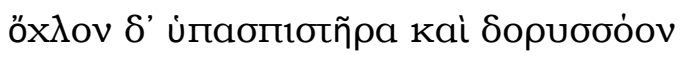

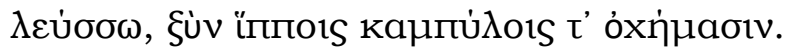

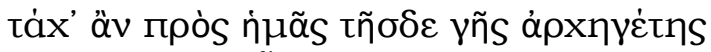

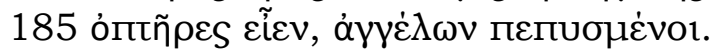

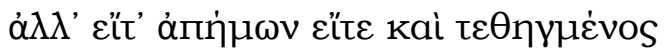

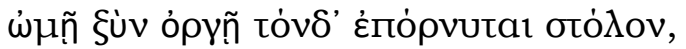

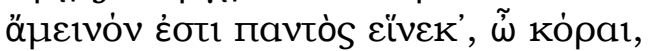

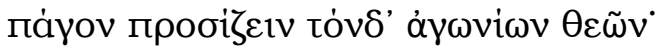

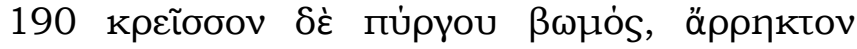
oákos.

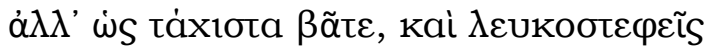

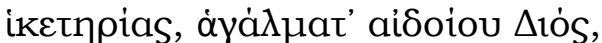

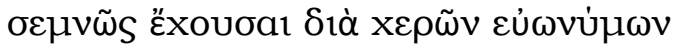

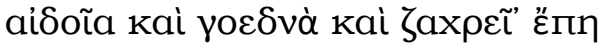

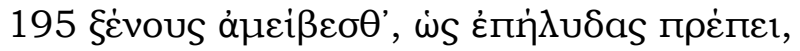

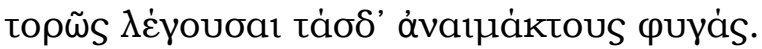
$\varphi \theta$

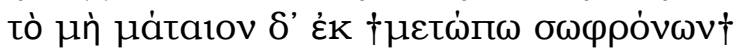
'เ

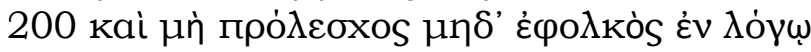

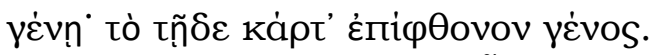

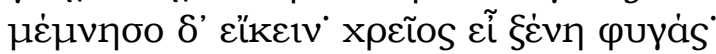

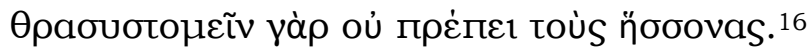

recomiendo que cuidéis estas palabras mías, como grabándolas en unas tablillas.

Estoy viendo polvo, silente mensajero del ejército; las siringas que giran sobre su eje no callan; pero brilla ante mis ojos una multitud que carga escudos y blande lanzas con caballos y curvos carros. Quizás venga hacia nosotros un gobernante de esta tierra [y sus seguidores] como observadores, porque a causa de la noticia de los mensajeros, quieren informarse.

Sin embargo, ya inofensivo, ya provocado por la salvaje cólera, excita a esta flota. Por todo esto es mejor, oh muchachas, que vengáis a sentaros en el promontorio de estos dioses que presiden la contienda. Pues un altar es más fuerte que una torre, escudo invulnerable.

No obstante, de esta manera, caminad lo más rápidamente posible $\mathrm{y}$, sosteniendo devotamente entre las prósperas manos ramas de olivo coronadas de blanco, ornamento en honor del piadoso Zeus, intercambiad con los huéspedes, como conviene a los recién llegados, no sólo llorosas, sino también impetuosas palabras, mientras relatáis claramente esta fuga no manchada por sangre. Pero, primero, por un lado, que la audacia no acompañe a la lengua; por el otro, que la insolencia no salga de los moderados rostros, de la pacífica mirada. Tampoco resultes pronta en hablar ni tediosa (lenta) en el discurso: la descendencia de aquí es extremadamente celosa.

Y recuerda ceder: eres necesitada, extranjera, fugitiva. Pues no conviene que los más débiles tengan una boca audaz. ${ }^{17}$

El segundo pasaje (710-733) sobreviene a continuación de la oda central (625-709), por lo tanto, inmediatamente después de la celebración inspirada por la confirmación del asilo. Nuevamente, dice Dánao:

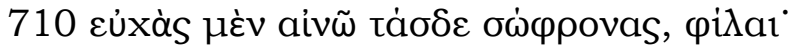

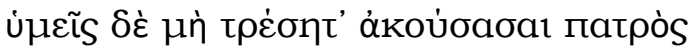

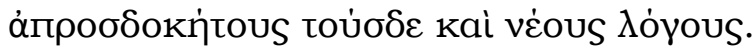

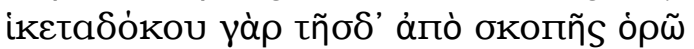

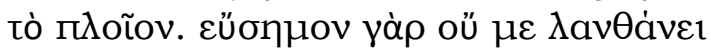

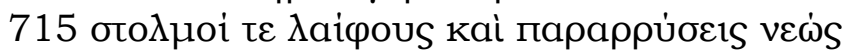

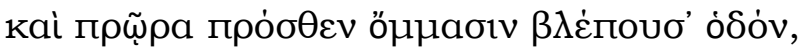

Por un lado, queridas, apruebo estos prudentes votos; por el otro, vosotras no temáis cuando escuchéis de su padre estas inesperadas y nuevas palabras.

Pues desde este atalaya de suplicante estoy viendo la embarcación, ya que es fácilmente reconocible: no se me ocultan

\footnotetext{
16 Las citas del texto original griego han sido tomadas de la edición de Sommerstein (2008).

${ }^{17}$ Las traducciones de ambos pasajes son propias.
} 


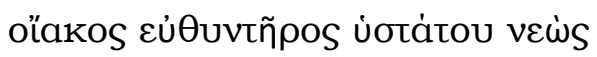

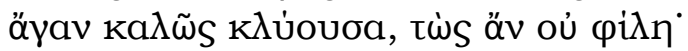

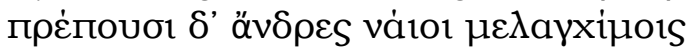

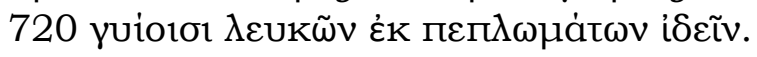

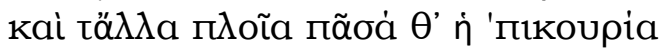

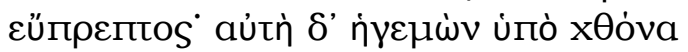

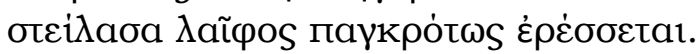

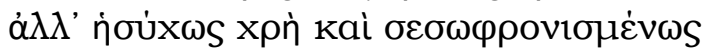

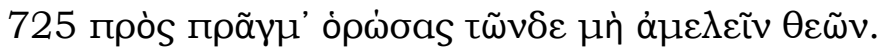

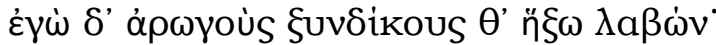

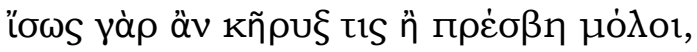

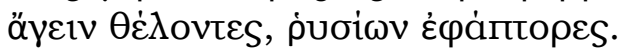

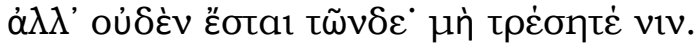

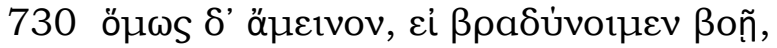

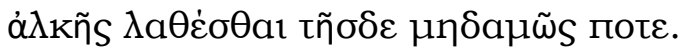

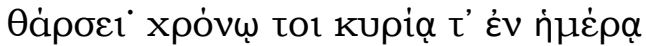

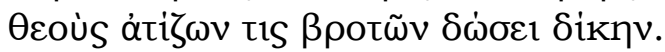

los equipamientos de lona ni los telones de la nave ni la proa en el frente que mira el camino con sus ojos mientras escucha demasiado bien al timón guía, como si no fuera amiga.

Y los varones parecen verse como marinos con negros miembros en contraste con sus blancos vestidos. También las otras embarcaciones y la fuerza auxiliar completa son conspicuas: pero esta, la conductora, después de amainar el velamen rema sincronizadamente, con mucho ruido, en dirección a tierra.

Sin embargo, es necesario que veáis este acontecimiento tranquila y prudentemente y que no descuidéis a los dioses: y yo volveré después de conseguir protectores y defensores. Pues probablemente venga algún heraldo o embajada, queriendo tomarlas, como raptores de sus botines. Pero no ocurrirá ninguna de estas cosas. Ahora no temáis, sino que es mejor, aunque eventualmente nos demoremos con el auxilio, no olvidarse de ninguna manera, en ningún momento, de esta defensa. Sé audaz: ciertamente, con el tiempo y en el día preciso cualquiera entre los mortales que desdeña a los dioses paga justicia.

La lectura correlativa de los discursos es iluminadora, pero los siguientes elementos consolidan la correspondencia y contraste entre ambos pasajes:

\section{Las invocaciones: vinculo entre hablante y destinatario}

El examen de las invocaciones es valioso porque expone una apreciación acerca de los destinatarios del discurso filtrada por la percepción subjetiva del hablante. Por lo tanto, proporciona datos del vínculo entre ambos. En la posición inicial del verso 176, паĩoss inaugura la primera intervención de Dánao en la obra. El sustantivo destaca su relación de parentesco y la dependencia de las jóvenes respecto de su padre. Como deíctico social, en un texto dramático sin acotaciones escénicas, el sustantivo identifica al emisor, someramente mencionado por las Danaides en el verso 11. Por eso, los versos 176-179 constituyen la presentación del personaje, que había ingresado junto al coro. ${ }^{18}$

En el verso 188, Dánao se dirige a las Danaides como kópat, que compendia una variedad de sentidos asociados: kópn es "hija"/"niña"/"virgen".19 Es interesante que la primera mención que se hace de las Danaides en la obra desde un punto de vista ajeno al de ellas mismas subraye el estatus social de las jóvenes. El vocativo es un recurso más que, desde el ámbito lingüístico, colabora en orientar al espectador respecto del matrimonio como uno de los tópicos fundamentales de la obra. No obstante el valor sociológico del término, se trata de un sustantivo que acentúa la

\footnotetext{
${ }^{18}$ Cfr. apartado II.2.1, "Participaciones I y II".

${ }^{19}$ LSJ : 980. El mismo sustantivo es utilizado para designar a Ártemis (145), invocada a propósito de su castidad. Bowen (2013: 184) destaca el sentido del término vinculado al estatus público de la joven aún no desposada.
} 
debilidad y desprotección de las Danaides, justificando la necesidad de la presencia de Dánao. Las referencias a su condición de inferioridad, que pueden ser rastreadas a lo largo de toda la obra, se retoman explícitamente entre los versos 202-203, final de este discurso. Por lo tanto, la rhêsis se sirve de una composición anular: el padre está presente, interviene y aconseja porque sus hijas son débiles.

En el segundo discurso, la invocación es pìaı (“queridas", 710), en la posición final del verso. Esta ubicación parece encadenar al vocativo con la frase siguente: Dánao sabe lo que va a anunciar, por eso, muestra contemplación y cariño. ${ }^{20}$ Según un criterio métrico, el espacio hubiera permitido otras posibilidades, como паи̃ठعs o kópai, elegidas antes. También en este caso, la invocación advierte acerca de una composición anular: los versos finales (732-733) insisten en la intención de Dánao de acompañar y dar ánimo a sus hijas.

En consecuencia, las invocaciones anticipan los tópicos principales de cada rhêsis, pues la primera tiene carácter preceptivo; la segunda es confortadora. Por lo tanto, muestran dos aspectos de la misma función de Dánao: en el primer caso, las protege como acompañante exigido por la debilidad e inexperiencia de las Danaides; en el segundo, como aquel que no sólo debe brindar seguridad efectiva, sino que debe transmitirla.

\section{E1 léxico verbal: las acciones esenciales de los discursos}

Mirar las rhéseis en conjunto permite distinguir fácilmente que ambas son introducidas por el mismo verbo, aivw̃ $(179 ; 710)$. En el primer discurso, al inicio del verso 179 , su sentido se perfila hacia el futuro, subrayando una acción en la que las Danaides deben ser activas: "cuidar" las palabras de su padre, actuando en consecuencia. Por eso, dentro de los matices semánticos que ofrece el verbo, ${ }^{21}$ se ha optado por interpretarlo como "recomendar". En el verso 710, en cambio, el mismo verbo dirige su atención hacia una acción ya realizada: el canto de las jóvenes dedicado a la ciudad de Argos, que les ha brindado protección. Este sentido pretérito es traducido como "aprobar". Acto seguido, Dánao propone a las Danaides una acción pasiva, escuchar. El empleo del verbo, más allá de su sentido prospectivo o retrospectivo, denota la autoridad de Dánao sobre la conducta de sus hijas.

Por otro lado, ambos discursos tienen origen en un acto de percepción sensible. Dánao goza de una posición privilegiada en lo más alto del altar y, porque puede ver, habla. ò $\tilde{\omega}$ ("estoy viendo") introduce la visión a distancia en la posición inicial del verso 180 en el primer discurso y, en el segundo, en el final del verso 713. $\mathrm{El}$ verbo tiene valor durativo 22 e implica, además, percepción inteligible. En palabras de Segal, "el pensamiento griego se inclina a considerar que la visión es el ámbito primario de conocimiento". ${ }^{23}$ En este caso, Dánao ve y, aunque no sabe con certeza, conjetura, lo que explica la construcción en el modo sintáctico potencial.

Asimismo, la comparación de las características del objeto de visión resulta esclarecedora. En el primer discurso, kóviv ("polvo", 180) es un sustantivo indeterminado que implica absoluta imprecisión respecto del referente que se describe a la distancia. El sustantivo que da mayor información está, en cambio, al final del verso, aludido de manera indirecta, en genitivo: orpatoũ ("del ejército", 180). En esta descripción visual, la metáfora del polvo como mensajero se completa con una imagen auditiva, metonímica: el sonido de los ejes de las ruedas insinúa la proximidad de los carros.

Coordinado por un $\delta \varepsilon \dot{\varepsilon}$ adversativo, se introduce en este primer discurso otro

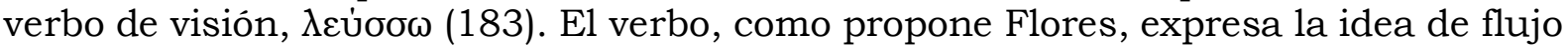
visual, de un hilo de luz que brilla desde los ojos hacia el objeto. ${ }^{24}$ En este caso, como

\footnotetext{
20 Bowen (2013: 184) señala que este vocativo posee fuertes connotaciones emocionales.

21 LSJ: 39.

22 Flores (2007: 79). Los matices semánticos han sido extraídos de Chantraine (1968: 813-815).

23 Segal (1995: 221).

${ }^{24}$ Flores (2007: 205). La noción de flujo es interpretada por la autora como "lanzar la mirada con los ojos."
} 
la percepción está vinculada con un objeto material que se destaca por su brillo, las armas, resulta adecuado traducir el verbo como "brillar ante los ojos". La selección léxica verbal no sólo pone el acento sobre el objeto visto, la multitud, sino que da cuenta de que, a medida que Dánao cuenta lo que ve, el objeto se acerca y se distingue mejor. Por eso, de este verbo depende la conjetura acerca de la identidad de quienes vienen (184-185).

De esta construcción sintáctica en modo potencial surge el predicativo

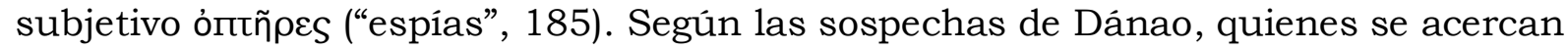
vienen para ver, es decir, para conocer. La relación entre los dos grupos, Danaides y Argivos, por lo tanto, es reciproca: necesitan verse para conocerse, más precisamente, "reconocerse". El anuncio de este acontecimiento fundamental para los protagonistas

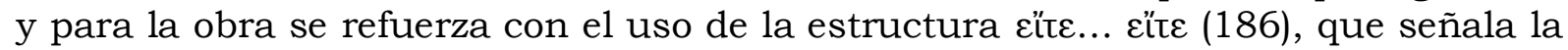
incertidumbre acerca del tipo de vínculo social objetivo que generará el encuentro.

En el segundo discurso, el objeto con el que se completa el sentido de óố aparece especialmente destacado por el encabalgamiento. A diferencia del caso anterior, tò пגoĩov ("la nave", 714) se presenta como sustantivo definido, característica morfosintáctica que destaca la condición particular de este otro referente que se describe a distancia: lo que Dánao divisa en esta oportunidad resulta familiar. En consecuencia, aquí no hay posibilidad de reconocimiento. El adjetivo

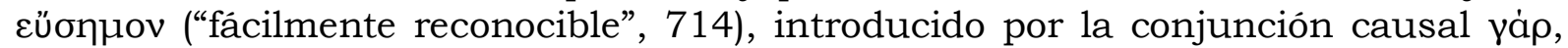
acentúa la oposición entre los objetos divisados. Entre 714 y 723, Dánao refiere todos los signos que confirman la identidad de quienes se aproximan. El verso final de esta descripción visual se asocia, también en este segundo anuncio, a otra imagen auditiva: el ruido producido por la sincronización y rapidez de los remos agrega tensión, insistiendo sobre la progresión del acercamiento (722-723).

A diferencia del primer anuncio, en esta oportunidad el verbo de percepción sensible se expande, como es adecuado al conocimiento del objeto, en un modo sintáctico real (714). Sin embargo, la potencialidad se instala luego de la descripción, ya que el padre debe justificar ante sus hijas que dispone de tiempo para ir en busca de ayuda. Por ello, en el verso 727, Dánao augura que "probablemente venga un heraldo o una embajada". Aunque la posición de este modo sintáctico no coincide con la del primer anuncio, la estructura potencial se amolda a la misma propuesta

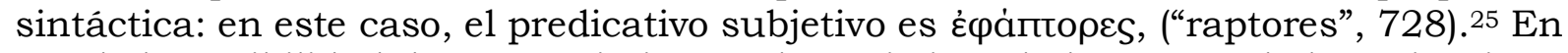
vez de la posibilidad de reconocimiento, el pasaje instala la certeza de la peripecia.

\section{Uso de la raiz Өapo-/ Өpao-: actitudes hacia quienes llegan 26}

El campo semántico que involucra esta raíz variable se aprovecha para dos

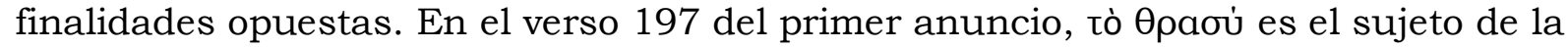
advertencia del padre a sus hijas antes de la llegada del potencial salvador: "que la audacia no acompañe a la lengua". En el mismo discurso, pocos versos después, Өpaбuбтонєі̃ (203) insiste en que: "no conviene que los más débiles tengan una boca audaz". Dánao, conocedor del pueblo receptor, aconseja a sus hijas moderación y sensatez, sobre todo al presentar su caso. En el verso 732 del segundo discurso, en cambio, la recomendación es inversa: con la fuerza del imperativo (Өápoє1, "sé audaz") Dánao alienta a sus hijas para que sean valientes, infundiéndoles ánimo para

\footnotetext{
${ }^{25}$ Cuzzi (1970: 85) señala la relación etimológica entre este sustantivo compuesto y Épafo, nacido de la ž (v. 46). Por lo tanto, los episodios de Zeus e Î́ y de los Egipcios y las Danaides se conectan también a través del uso de las palabras, en este caso, coincidiendo en la idea de la mujer como objeto de posesión masculina.

${ }^{26}$ Respecto de la coincidencia de ambos anuncios en el empleo de otras raíces semánticas importantes, vale la pena

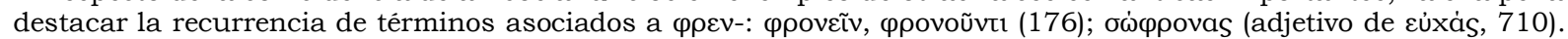
En el primer caso, Dánao reconoce aquello que las Danaides han dicho de él al mencionarlo (11) y, al mismo tiempo, lo recuerda a sus hijas como el modo adecuado de comportamiento. En el segundo discurso, el padre elogia el accionar correcto de sus hijas, que han cantado en agradecimiento a la ciudad benefactora. El campo semántico se retoma en el discurso final de Dánao, justamente en la última línea que profiere el actor-carácter (992-2013). Por lo tanto, los discursos de Dánao se ajustan al patrón de composición anular, frecuente en la obra en distintos niveles de análisis.
} 
enfrentar a sus perseguidores. ${ }^{27}$ En sintesis, apocamiento frente al huésped para ser aceptados como amigos; arrojo frente al raptor, que ya es enemigo.

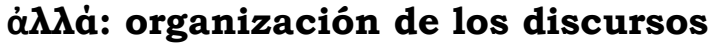

La partícula adversativa divide ambos discursos en dos y retoma, luego de los anuncios, el sentido inicial: la recomendación. Tras la descripción de la visión a la distancia, à $\lambda \lambda \dot{\alpha}(191 ; 724)$ inaugura la cadena de imperativos. ${ }^{28}$ En el primer anuncio,

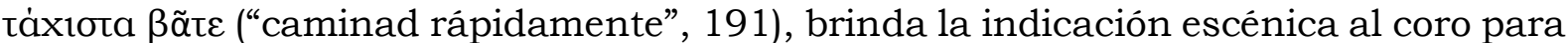
que se posicione junto a los altares de los dioses, que les brindan inmunidad. Le siguen otras directrices vinculadas con las convenciones griegas de la súplica: el uso de los ramos, la forma, y el gesto que resultan adecuados para hablar al huésped.

De manera idéntica, en el verso 724 , la misma partícula introduce una pauta de acción en función de lo que se ha visto: $\tau \tilde{\omega} v \delta \varepsilon \mu \eta \dot{\alpha} \mu \varepsilon \lambda \varepsilon \tilde{v} v \theta \varepsilon \tilde{\omega} v$ ("no descuidéis a estos dioses", 725). El consejo es, entonces, permanecer al mismo resguardo.

\section{Modos sintácticos y otras construcciones sintácticas: el rol de Dánao}

Como ha sido señalado, la conjunción adversativa $(186 ; 724)$ divide las rhéseis en dos. Sin embargo, ambas partes colaboran para convertirse en un motor generador de palabras y acciones: la primera parte de ambos discursos involucra la anticipación de la posibilidad de salvación o de la proximidad del peligro y está dominada por el modo sintáctico real. La inquietante cercanía de quienes llegan conduce, en acelerado in crescendo, a la yuxtaposición de los numerosos imperativos y prohibitivos de la segunda parte. El modo sintáctico real de los anuncios sólo es invadido por el potencial de los versos 184 y 727, cuando Dánao conjetura quiénes pueden ser aquellos que ve a distancia.

Las gnômai que profiere Dánao como sensato anciano (176-179) brindan otra oportunidad para analizar el paralelismo trazado por estos dos discursos. En el verso 190, el padre afirma que "un altar es más fuerte que una torre, escudo invulnerable"; entre los versos 732-733, declara que "ciertamente, con el tiempo y en el día preciso, cualquiera entre los mortales que desdeña a los dioses paga justicia". Ambas máximas exponen el juego de oposición entre las Danaides y los hijos de Egipto. ${ }^{29}$ Las "recién llegadas" y, en parte, Argivas, se aproximan a los ideales griegos de la sofros $\_$ne y la piedad debida a los dioses. En cambio, sus primos, caracterizados permanentemente como hybristai e impíos, merecen ser identificados como "bárbaros".

También en consonancia con la sabiduría de Dánao, la construcción nominal

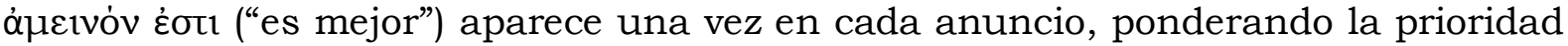
en cada una de las coyunturas. En el verso 188, Dánao dice: "es mejor, por todo esto, que vengáis a sentaros en el promontorio de estos dioses que presiden la contienda". Frente a la presión que instaura la llegada del potencial protector, Dánao manifiesta la necesidad de conseguir el cobijo de las divinidades. Por consiguiente, en la frase se destacan los deícticos de movimiento o dirección. Asimismo, es clave el adjetivo que califica a los dioses, $\dot{\alpha} \gamma \omega v i \omega v$, derivado de agón: se trata de los dioses que presiden cualquier reunión entre personas, ya sea un encuentro cooperativo como uno competitivo.

\footnotetext{
27 La audacia es un componente obligatorio de la personalidad del héroe épico. Por lo tanto, Dánao estaría demandando a las Danaides no sólo una conducta heroica, sino también masculina.

28 Según la clasificación de Denniston (1954: 13-14), este uso de à $\lambda \lambda$ á en ambos discursos parece ajustarse a un uso especial, propio de las órdenes y exhortaciones: "it rather expresses, as Hartung says (ii35), a break off in the thought or, as Klotz (i5) more specifically and more accurately puts it, a transition from arguments for action to a statement of the action required. Hence $\dot{\alpha} \lambda \lambda \dot{\alpha}$, in this sense, usually occurs near the end of a speech, as a clinching and final appeal (whereas at the opening of a speech it introduces and objection in the form of a command: S. El. 431 'Nay'): as we say, 'Oh, but do', 'come' or 'come now' will often get the meaning”. La última sugerencia ha sido la adoptada en nuestra traducción del pasaje.

${ }^{29}$ Cabe destacar que, en ambos discursos, Dánao recurre a las sentencias sobre el final.
} 
En el verso 730 del segundo discurso, Dánao formula: "es mejor, aunque eventualmente nos demoremos en el auxilio, no olvidarse, de ninguna manera, en ningún momento, de esta defensa". Si bien las Danaides no se instalan inmediatamente en el montículo del altar desde donde Dánao divisa a los enemigos, aprovechan la protección del recinto sagrado tras la llegada de Pelasgo (911).

Tras este análisis, es irrefutable la composición estructural de la obra sobre la base de escenas espejadas. Resulta evidente el paralelismo entre el comienzo y el "nuevo comienzo" que se instala tras el segundo discurso. La correspondencia escénica marca dos hitos en la obra, que diseñan un esquema compositivo en el cual los acontecimientos definitorios son las llegadas de los personajes hasta el lugar donde permanece el coro protagonista. Los movimientos, entonces, delinean las partes de la pieza.

El doble uso del recurso del anuncio extendido se justifica a partir de la marca convencional que signa la tragedia y le da su nombre: las reglas de la hiketeía obligan a todo suplicante a llevar a cabo determinados comportamientos, socialmente establecidos, para asegurarse la protección deseada. Por lo tanto, ambos discursos obedecen tanto a una necesidad escénica como dramática. En cuanto a su función escénica, dan tiempo a las Danaides para ubicarse en el lugar y posición que, según Dánao, les corresponde en su carácter de suplicantes: postrarse a los pies de las estatuas. Esta actitud escénica y, sobre todo, la función de Dánao como chóregos, son más evidentes en el primer anuncio, ya que se trata de la primera vez que se explican estas convenciones griegas. En el segundo caso, las indicaciones se reiteran,

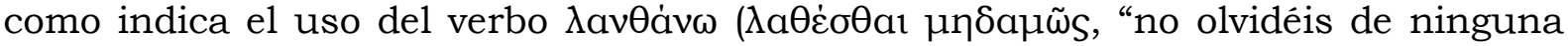
manera", 731). No obstante, la segunda escena marca una diferencia en la reacción de las Danaides: la demora en la toma de posición en el altar permite generar la tensión del encuentro entre las jóvenes y el heraldo en el espacio de la orchéstra (825 y ss.)

Respecto de la motivación dramática, la composición alrededor de los anuncios demuestra que Suplicantes no es, como se ha sostenido tradicionalmente, una tragedia "simple". Los anuncios que forman parte de estos dos discursos dan cuenta de la madurez de la obra porque, además de constituir un dispositivo arquitectónico, funcionan como anticipación de las metabolaí, de los cambios, que son las acciones más complejas que puede presentar una tragedia según el criterio clásico aristotélico. Desde el primer discurso de Dánao, coro y público palpitan el encuentro con los Argivos y viven con expectativa el reconocimiento, es decir, si de ese encuentro resultará una relación de alianza u hostilidad. ${ }^{30}$ Luego ambos personajes descubren algo que antes ignoraban, que están ligados entre sí por dos relaciones objetivas socialmente definida como positivas: por un lado, la philía determinada por el vínculo de parentesco; por el otro, el vínculo de cooperación que asocia a suplicante y suplicando. A partir del momento en que Dánao pronuncia el segundo discurso de anuncio, el efecto sorpresa genera, en cambio, que Danaides y espectadores comiencen a experimentar la peripecia, que se materializa con la llegada del heraldo de los Egipcios (825).

Desde el criterio moderno, que pondera la calidad dramática de las obras según la presencia de conflicto, los anuncios extendidos también introducen los dos encuentros agonales fundamentales, determinados por el motivo de súplica: el agón

\footnotetext{
30 Seguimos la propuesta de Dupont- Roc y Lallot (1980: 232-233, n. 2), quienes interpretan la definición aristotélica de "reconocimiento" (Poética 1452 2 29) de la siguiente manera: "Philía désigne le lien qui unit les membres d'un groupe clos, et en particulier le lien de parenté ou d'alliance (...); symétriquement, l'ekhthrá désigne 1'hostilité de fait qui découle, notamment, de la violation d'un tel lien. Moins que l'appréhension subjective que le héros peut avoir de son action ou des ses relations à l'autre, la reconnaisance est la découverte du fait, ignoré de lui auparavant, qu'il est lié à tel autre personnage par une relation objective, socialement définie comme positive (PHILÎA) ou négative (ÉKHTHRA): ainsi Oedipe qui 'reconnaît la PHILÍA' qui l'unit à son père". La cita manifiesta que, en este tipo de cambio de fortuna, el meollo no es simplemente el conocimiento de la identidad de un personaje, sino la toma de conciencia del tipo de vínculo social que existe entre un personaje y otro. Este dato es clave para la aproximación a las tragedias de súplica.
} 
entre el suplicante y el potencial salvador, para obtener la protección necesaria, y el agón con el perseguidor, para intentar rechazar su dominio.

En sintesis, los dos discursos analizados no solamente anticipan las llegadas de los nuevos caracteres, Pelasgo y el heraldo, que es lo que indica la percepción sensible, sino los cambios que se producirán en el acontecimiento trágico. Los anuncios, por lo tanto, construyen los momentos de mayor clímax dramático y dividen la obra en dos mitades.

Los prejuicios sobre el primitivismo de Suplicantes estaban basados en la incapacidad de comprender la forma de un drama que resulta poco habitual. Sin duda, es natural, pero errado, aproximarse a una obra de arte con una idea previa de lo que debe ser. Efectivamente, estamos convencidos de que, como críticos, debemos cuestionar fundamentalmente aquello que percibimos como irregular, puesto que, cuando un artista no recurre al procedimiento obvio, es lógico pensar que pretenda alcanzar otro efecto, uno en el que el recurso esperable hubiera fracasado.

El análisis de los anuncios extendidos ha permitido acercarnos a la forma de esta obra en particular, sin atender a ningún molde preestablecido, normado y artificial. La simetría estructural de la obra generada por estos pasajes no sólo funciona como dispositivo arquitectónico gracias a su especularidad, sino también como recurso de preparación, ya que anticipa las escenas de metabolaí y de agón, es decir, las de mayor tensión dramática.

En este punto, resulta pertinente volver sobre el epígrafe que dio inicio a este trabajo, porque los textos de la mayoría de las obras trágicas continúan siendo introducidos, tal como en las antiguas hipótesis, por un resumen del argumento. Esta desafortunada tradición ha naturalizado el hecho de que la tragedia griega sea reducida a un exiguo párrafo en un sinnúnero de manuales modernos. ${ }^{31}$ Sin embargo, una sintesis argumental no dice nada acerca las cualidades especiales y puntos de énfasis de una obra. Por ello, los anuncios extendidos resultan un ejemplo que logra demostrar la relación entre la obra en papel y la acción en el escenario, dentro de las condiciones y posibilidades de realización de la tragedia griega clásica. De su estudio emergieron dos valiosas conclusiones: 1. que es posible recuperar de algún modo la puesta en escena que el dramaturgo concibió y presentó como parte de su creación, considerando las "acciones significativas" como aquellas especialmente subrayadas por las palabras; 2. que la forma de la trama no es una característica externa ni incidental, sino una parte de la composición artística, un elemento de comunicación y, por lo tanto, una clave de sentido.

En definitiva, aprehender un aspecto de la forma compositiva particular de Suplicantes ha revelado cómo la tragedia provoca el efecto dramático cuando logra volver apasionantes los movimientos de llegada de los actores hasta el sitio ocupado por el coro protagonista, manipulando con extraordinaria pericia los momentos de sorpresa y expectación.

\section{Referências Bibliográficas}

BOWEN, A. J. (2013) Aeschylus Suppliant Women. Edited with a translation, Introduction and commentary, Oxford. ChANTRAINe, V. (1968) Dictionnaire étymologique de la langue grecque, Paris.

\footnotetext{
31 Taplin (1977: 26).
} 
Cuzzi, E. (1970) Studio sui composti delle Supplici di Eschilo, Milano.

DENNISTON, J. D. (1954) The Greek Particles, Oxford.

Dupont-Roc, R. ET LALlot, J. (1980) Aristote. La Poétique. Texte, traduction, notes, Paris.

FERNÁNDEZ DEAGUSTINI, M. DEL P. (2015) Suplicantes de Esquilo. Una interpretación (tesis doctoral), UNLP, http://hdl.handle.net/10915/51947.

FLORES, E. (2007) Esquilo. Aristeia de los ojos en la tragedia griega, San Juan.

GARVIE, A. (2006) Aeschylus' Supplices. Play and Trilogy (corrected ed.), UK.

LIDELL, H. G-SCOTT, R. (1996) A Greek- English Lexicon, Oxford.

Lobel, E. -Wegener, E. P.- RoberTs, C. H. (1952) The Oxyrinchus Papyri. Part XX $\left(\mathrm{N}^{\circ}\right.$ s. 2245-2287), London.

SEGAL, CH. (1995) "El espectador y el oyente" en VERNANT, J. (ed.) El hombre griego, Madrid: 211-246.

Sommerstein, A. H. (2008) Aeschylus. Persians, Seven against Thebes, Suppliants, Prometheus Bound, Cambridge, Massachusetts, London.

TAPLIN, O. (1977) The Stagecraft of Aeschylus. The Dramatic Use of Exits and Entrances in Greek Tragedy, Oxford.

---------- $(1978,2003)$ Greek Tragedy in Action, London and New York.

Wolf, E. A. (1958) "The date of Aeschylus' Danaid tetralogy", Eranos 56.3-4: 119139.

YORKE, E. C. (1954), "The date of the Supplices of Aeschylus", CR 4.1: 10-11. 